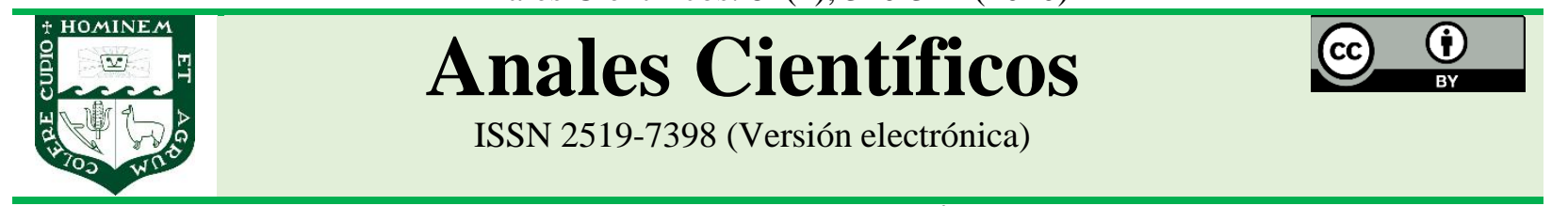

ARTÍCULO ORIGINAL - RESEARCH ARTICLE

http://dx.doi.org/10.21704/ac.v81i2.1642

\title{
OCURRENCIA ESTACIONAL DE PLAGAS Y PREDADORES EN EL CULTIVO DE SANDIA (Citrullus lanatus) (Thunb.) EN LA MOLINA
}

\section{Seasonal occurrence of pests and predators in growing watermelon (Citrullus lanatus) (Thunb.) in La Molina}

\author{
Daniel Ccahuana ${ }^{1}$; Germán Joyo ${ }^{1 *(\mathbb{D})}$; Jorge Castillo ${ }^{1}$; Alfredo Beyer ${ }^{1}$; \\ Patricia Rodriguez ${ }^{1}$ \\ ${ }^{1}$ Facultad de Agronomía, Universidad Nacional Agraria La Molina, 15024, Lima, Perú. \\ *E-mail: gjoyo@lamolina.edu.pe
}

Recibido: 20/06/2019; Aceptado: 15/12/2020; Publicado: 30/12/2020

\begin{abstract}
Observations were made to determine the seasonal occurrence of Diaphania nitidalis (Stoll), Prodiplosis longifila (Gagne), Liriomyza huidobrensis (Blanchard), Bemisia tabaci (Gennadius), Aphis gossypii (Glover), Agrotis ipsilon (Hufn.) Spodoptera ochrea (Hampson) and their natural enemies in watermelon's crop. located in the Agrarian University of La Molina were made the land called Field Free I of 1 ha. It was divided into five sectors in which were taken randomly 5 plants per sector, there were evaluated 25 plants in total. On each plant four outbreaks were recorded, 4 sheets randomly, as the foliage of the plant, 4 flowers and 4 fruits distributed throughout the plant, the neck of the plant and 2 meters of furrow by sector. The earth worm Agrotys ipsilon (Lepidoptera: Noctuidae) did not cause major damage. As for Diaphania nitidalis (Stoll) (Lepidoptera: Crambidae) infestation was low in the first months of the crop (January, February and March); however, in the month of April they increased records of individuals in buds, flowers and fruits. It should be noted the presence of Spodoptera ochrea (Hampson) (Lepidoptera: Noctuidae) who did affect significantly during the stages of vegetative growth, flowering and fruiting; infesting leaves, flowers and fruits. They were registered as natural enemies Chrysoperla externa (Hagen) and Cycloneda sanguinea (Linnaeus).
\end{abstract}

Keywords: seasonal occurrence; Diaphania nitidalis; Prodiplosis longifila; Liriomyza huidobrensis; Bemisia tabaci; Aphis gossypii; Agrotis ipsilon; Spodoptera ochrea; watermelon; shoots; leaves; flowers; fruits; natural enemies

\section{RESUMEN}

Se realizaron observaciones con el objetivo de determinar la ocurrencia estacional de Diaphania nitidalis (Stoll), Prodiplosis longifila (Gagné), Liriomyza huidobrensis (Blanchard), Bemisia tabaci (Gennadius), Aphis gossypii (Glover), Agrotis ipsilon (Hufn.), Spodóptera ochrea (Hampson) y sus enemigos naturales en el cultivo de sandía. El estudio se realizó en el área Campo Libres I de la Universidad Nacional Agraria La Molina. La parcela fue de una hectárea en total, la evaluación se hizo dividiendo el campo en cinco sectores evaluando cinco plantas en cada sector, 25 plantas en total. Por planta se evaluó cuatro brotes, cuatro hojas aleatoriamente, cuatro flores y cuatro frutos a lo largo de la planta, el cuello de planta y dos metros lineales de surco en cada sector. Agrotys ipsilon 
(Lepidóptera: Noctuidae) no fue un problema serio. Respecto a Diaphania nitidalis (Stoll) (Lepidoptera: Crambidae) el ataque fue menor los tres primeros meses del cultivo; sin embargo, al cuarto mes en abril se incrementó su presencia en brotes, flores y frutos. Destacó Spodoptera ochrea (Hampson) (Lepidóptera: Noctuidae), que infringió un daño importante en desarrollo vegetativo, floración y fructificación; afectando hojas, flores y frutos. Los controladores biológicos encontrados fueron Chrysoperla externa (Hagen) y Cycloneda sanguínea (Linnaeus).

Palabras clave: ocurrencia estacional; Diaphania nitidalis; Prodiplosis longifila; Liriomyza huidobrensis; Bemisia tabaco; Aphis gossypii; Agrotis ìpsilon; Spodóptera ochrea; sandía; brotes; hojas; flores; frutos; enemigos naturales

\footnotetext{
Forma de citar el artículo (Formato APA): cultivo de sandía (Citrullus lanatus) (Thunb.) en La Molina. Anales Científicos. 81(2), 310-321.

http://dx.doi.org/10.21704/ac.v81i2.1642

Autor de correspondencia (*): Germán Joyo. Email: gjoyo@lamolina.edu.pe

(c) Los autores. Publicado por la Universidad Nacional Agraria La Molina.

This is an open access article under the CC BY
}

Ccahuana, D., Joyo, G., Castillo, J., Beyer, A., \& Rodriguez, P. (2020). Ocurrencia estacional de plagas y predadores en el

\section{INTRODUCCIÓN}

La sandía (Citrullus lanatus (Thunb)) es una de las hortalizas más cultivadas en el mundo (Huh et al., 2008). Es la cucurbitácea más consumida en el mundo. Representa el 6,8\% del área mundial dedicada a la producción de hortalizas (Guner y Wehner, 2004; Goreta et al., 2005).

La costa del Perú presenta condiciones edafoclimáticas favorables para el cultivo de hortalizas y frutas; siendo la sandía una de ellas, además de melones, fresas, moras o frambuesas; las cuales tiene potencial de exportación (Zohary y Hopf, 2000).

Las principales plagas de la sandía son Diaphania nitidalis (Stoll), Prodiplosis longifila (Gagné), Liriomyza huidobrensis (Blanchard), Bemisia tabaci (Gennadius), Aphis gossypii (Glover), Agrotis ipsilon (Hufn.), Agrotis malefida (Guen.), Peridroma saucia (Hubn.) y Feltia experta (Walker), sin embargo, existen en este cultivo diversos predadores y parasitoides que regulan la población de estos fitófagos (Escalona et al., 2009).

La investigación evaluó y monitoreó la presencia de plagas y enemigos naturales en el cultivo de sandía (Citrullus lanatus) (Thunb), en La Molina.

\section{MATERIALES Y MÉTODOS}

El presente trabajo de investigación se llevó a cabo en el lote denominado Campo Libres I, teniendo un área aproximada de 1 ha, ubicado en el área agrícola de la Universidad Nacional Agraria La Molina, distrito de La Molina, Provincia de Lima, Departamento de Lima, Perú. Las evaluaciones tuvieron lugar de diciembre de 2014 hasta el mes de abril de 2015.

La evaluación de plagas y enemigos naturales se llevó a cabo en un área aproximada de una hectárea, subdividiendo el área en cinco sectores, evaluando cinco plantas en cada sector, sumando 25 plantas en total. Por planta se revisó: cuatro brotes o una yema terminal, cuatro hojas al azar, cuatro flores, cuatro frutos por planta y $2 \mathrm{~m}$ lineales de surco por sector.

El ingeniero Walter Apaza proporcionó los datos de temperatura máxima $\left({ }^{\circ} \mathrm{C}\right)$, temperatura mínima $\left.{ }^{\circ}\right) \mathrm{C}$ ) y humedad relativa promedio (\%), medidos mediante una estación meteorológica automática a su cargo, ubicada a $12{ }^{\prime} 05^{\circ}$ latitud sur, $766^{\prime} 57^{\circ}$ longitud oeste y a 238 m.s.n.m. de la Universidad Nacional Agraria La Molina comprendidos entre diciembre del 2014 hasta abril del 2015.

\section{RESULTADOS Y DISCUSIÓN}

\section{Agrotis ipsilon (Hufnagel)}

En la Figura 1, se aprecia el ataque de Agrotis ipsilon luego del trasplante en el periodo vegetativo. El 31 de diciembre de 2014 alcanzó una densidad poblacional máxima de 4 larvas en diez metros lineales, a una temperatura de $20,06^{\circ} \mathrm{C}$, y con humedad relativa de $87,32 \%$. El 7 de enero de 2015 presentó únicamente 1 larva en diez metros lineales, a $20,58^{\circ} \mathrm{C}$, y con humedad relativa de $85,52 \%$. 
Las larvas de Agrotis ipsilon (Hufn.) causaron pérdidas de plantas en las dos primeras semanas seguidas al trasplante. De este modo, el 31 de diciembre tuvo lugar un máximo de $20 \%$ de plantas cortadas, y $5 \%$ el 7 de enero del 2015 en la segunda evaluación. La planta es más susceptible al momento del trasplante dado que al proseguir el crecimiento el tallo se endurece y se vuelve más resistente al corte de las larvas.

\section{Diaphania nitidalis (Stoll)}

De acuerdo a la Figura 2, la única fecha que se registró huevos por brotes de Diaphania nitidalis (Stoll), fue la evaluación del 31 de diciembre registrando 2 huevos a $20,06^{\circ} \mathrm{C}$ y humedad relativa de $87,32 \%$. El ataque de larvas en brotes, recién se presenta en la segunda evaluación el 07 de enero, registrando tres larvas a $20,58^{\circ} \mathrm{C}$ y $85,52 \%$ de humedad relativa. No se registró larvas el 11 de febrero y el 25 de marzo de 2015 debido a la aplicación de metomil; sin embargo, se registró su presencia en las evaluaciones del 08 y 15 de abril, presentando en ambos casos 6 larvas a $22,03^{\circ} \mathrm{C}$ y $23,3^{\circ} \mathrm{C}$ respectivamente y el máximo fue en la evaluación del 22 de abril al contabilizarse 9 larvas a $20,12^{\circ} \mathrm{C}$ y humedad relativa de $86,77 \%$.

La Figura 3 muestra el número de larvas en 100 flores y el número de flores afectadas, registrando 5 larvas y 7 flores infestadas en la única y última fecha de evaluación del 22 de abril, a $20,12^{\circ} \mathrm{C}$ y humedad relativa de $86,77 \%$.

La aplicación del insecticida metomil influyó en la ocurrencia estacional de Diaphania nitidalis. Zarate (1972), estableció en su ensayo comparativo en el cultivo de pepinillo donde probó insecticidas clorados como Parathión y Tamarón, carbamatos como Lannate y Furadán, encontrando que las pulverizaciones con Lannate 90 PM (metomil) al $0,1 \%$ proporcionó un resultado satisfactorio para el control de Diaphania nitidalis.

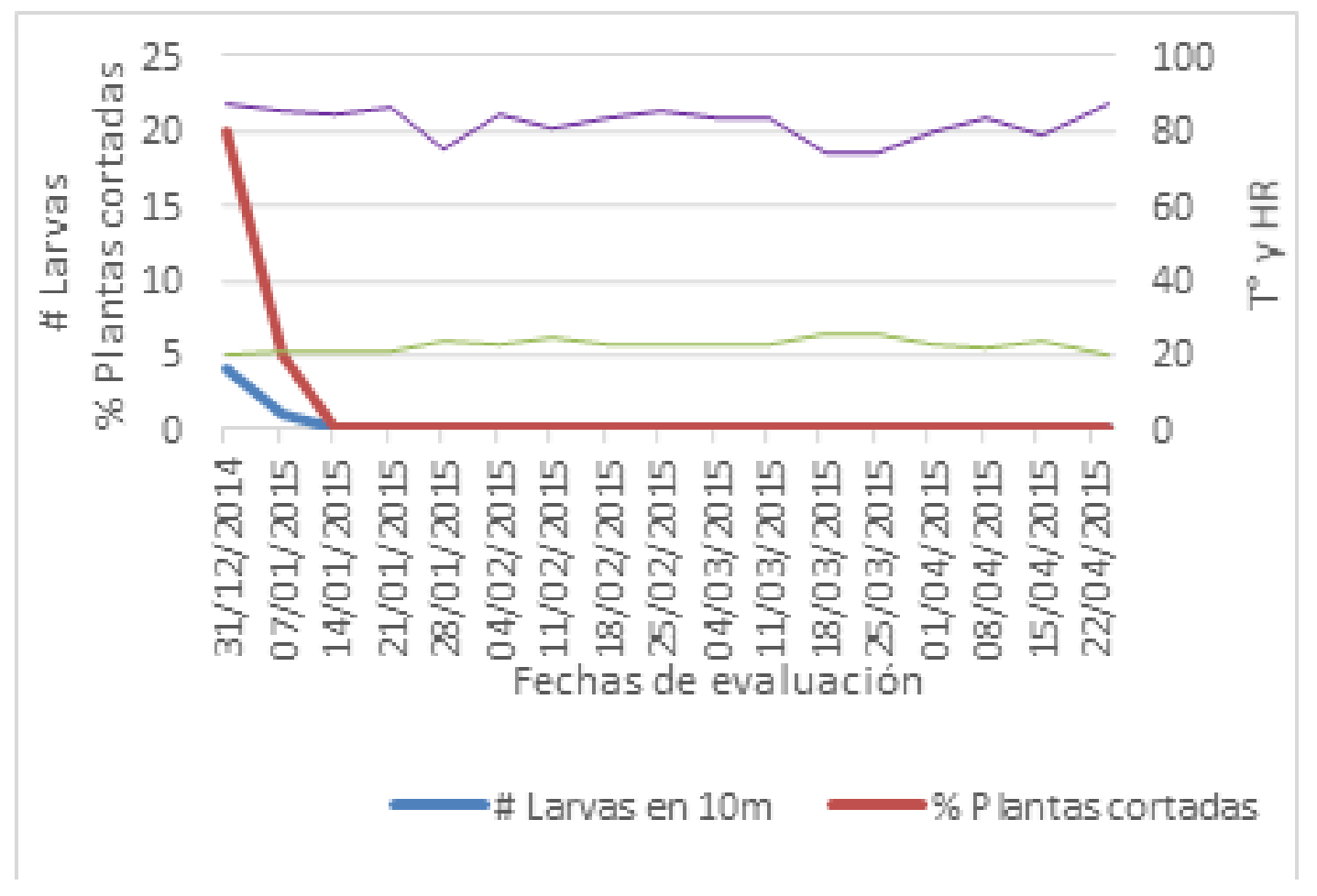

Figura 1. Ocurrencia estacional e larvas de Agrotis ípsilon (Hufn.) y porcentaje de plantas cortadas en sandía, variedadSanta Amelia. Periodo: diciembre 2014-abril 2015. La Molina,Lima-Perú. 

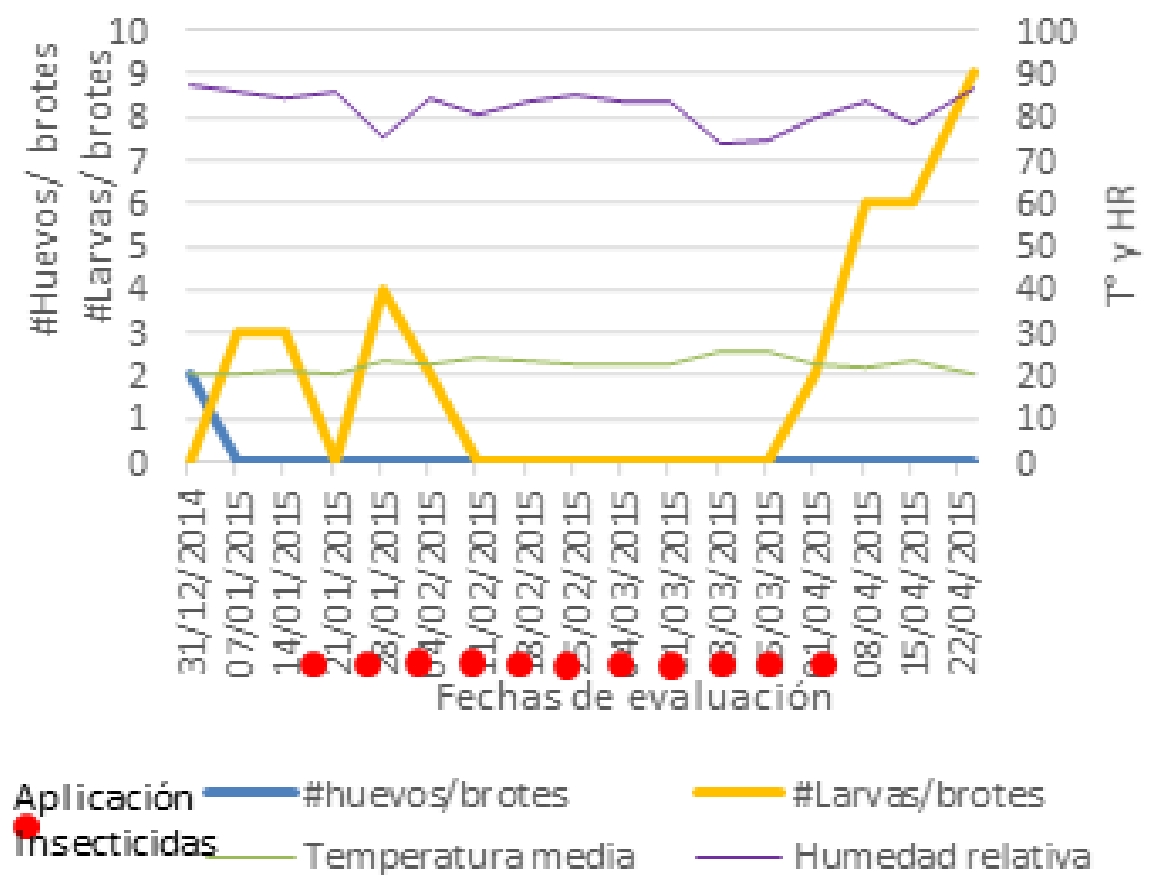

Figura 2. Ocurrencia estacional del número de huevos y larvasde Diaphania nitidalis (Stoll) en 100 brotes de sandía, variedadSanta Amelia. Periodo: diciembre 2014 - abril 2015. La Molina,Lima - Perú.

Esta plaga se registra a partir del 01 de abril con 3 larvas y 3 frutos afectados a $22,95^{\circ} \mathrm{C}$ y humedad relativa de 79,68\%; para luego mostrar un incremento en todo el mes de abril y alcanzar su máxima densidad poblacional el 22 de abril del 2015, con 11 larvas en frutos y 11 frutos infestados a $20,12{ }^{\circ} \mathrm{C}$ de temperatura y $86,77 \%$ de humedad relativa, como se aprecia en la Figura 4. Dicho incremento poblacional puede tener relación con la suspensión de la aplicación de insecticidas.

Diaphania nitidalis (Stoll) afectó desde el desarrollo vegetativo infestando brotes hasta el crecimiento del fruto. Sobre el particular, Sánchez y Vergara (2003) indican que las larvas perforan y barrenan yemas, flores, tallos y frutos y expulsan sus excrementos dejándolos en el exterior. En cuanto a los frutos infestados, los pequeños caen, y los más desarrollados se pudren y ya no son aptos para el mercado.

\section{Liriomyza huidobrensis (Blanchard)}

La Figura 5 muestra que la única evaluación con 1 adulto registrado por cien hojas evaluadas fue la del día 07 de enero, a $20,58{ }^{\circ} \mathrm{C}$ y con humedad relativa de $85,52 \%$.

En cuanto a hojas infestadas por Liriomyza huidobrensis, el 21 de enero se registró 6 hojas infestadas a $20,62^{\circ} \mathrm{C}$ y $86,12 \%$ de humedad relativa y el 28 de enero se registró 8 hojas a $23,3^{\circ} \mathrm{C}$ y con $75,14 \%$ de humedad relativa durante el desarrollo vegetativo.

No hubo registro de Liriomyza huidobrensis desde febrero a causa de las aplicaciones semanales de metomil; tal como indica Mujica y Cisneros (1995), el control químico es el método más empleado a través del uso de larvicidas y adulticidas de Liriomyza huidobrensis (Blanchard). 


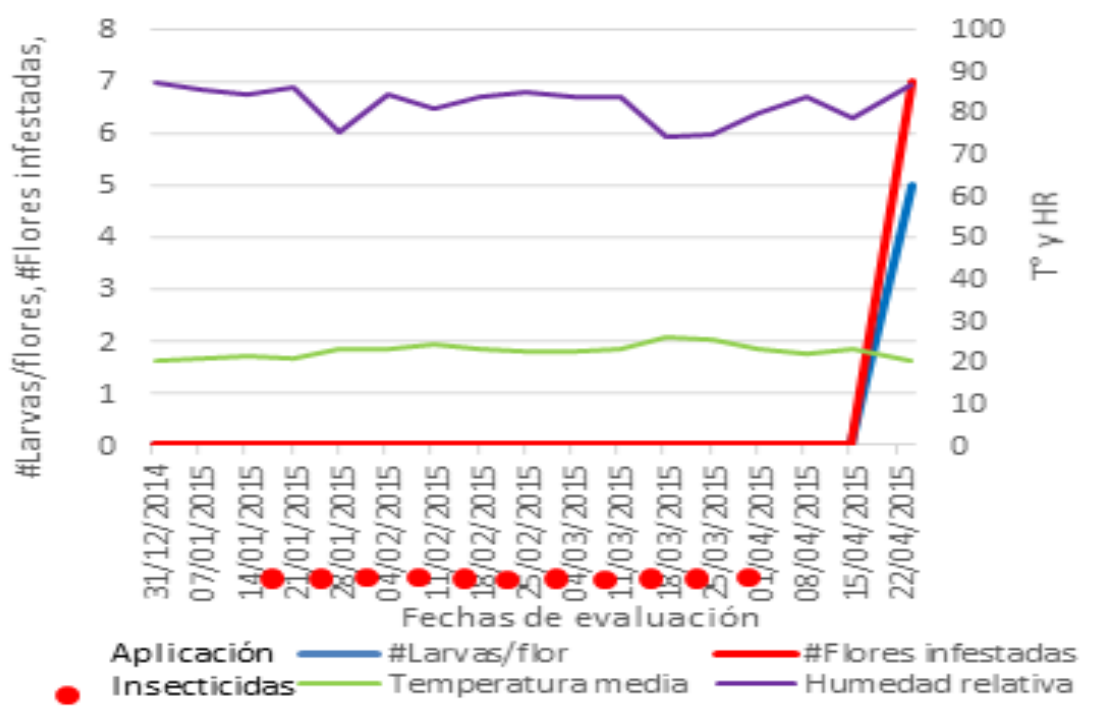

Figura 3. Ocurrencia estacional de larvas de Diaphania nitidalis (Stoll) en 100 flores y número de flores infestadas en sandía, variedad Santa Amelia. Periodo: diciembre 2014 - abril 2015. La Molina, Lima - Perú.

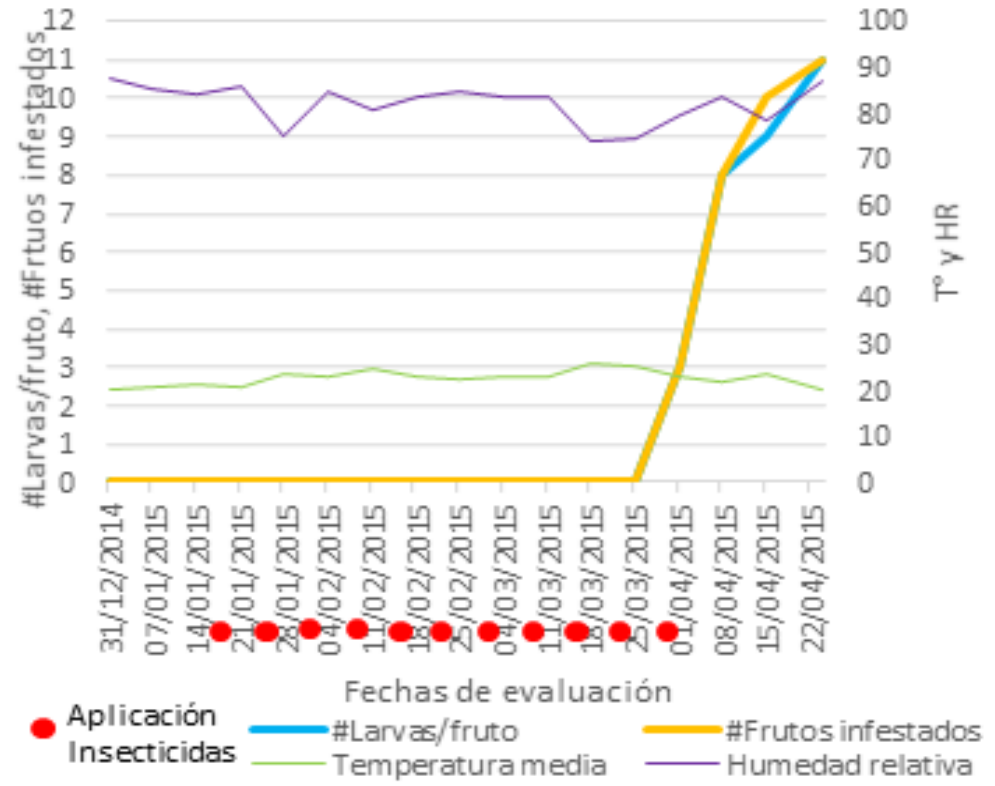

Figura 4. Ocurrencia estacional de larvas de Diaphania nitidalis (Stoll) en 100 frutos y número de frutos infestados en sandía, variedad Santa Amelia. Periodo: diciembre 2014 - abril 2015. 


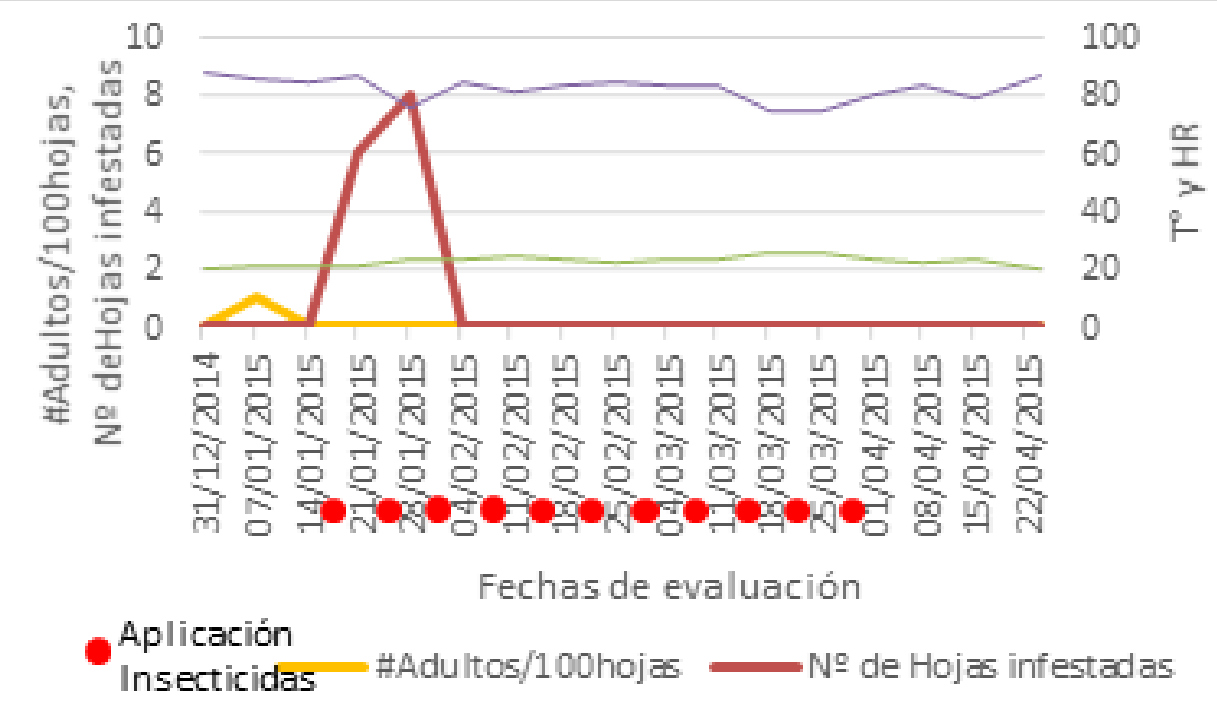

Figura 5. Ocurrencia estacional de adultos de Liriomyza huidobrensis(Blanchard) en 100 hojas y número de hojas infestadas en sandía, variedad Santa Amelia. Periodo: diciembre 2014 - abril 2015. La Molina, Lima - Perú.

\section{Aphis gossypii (Glover)}

La Figura 6 indica la presencia de 4 individuos el 07 de enero a $20,58^{\circ} \mathrm{C}$ y humedad relativa de $85,52 \%$. El 21 de enero tocó su punto más bajo de población registrando 1 individuo a $20,62^{\circ} \mathrm{C}$ y humedad relativa de $86,12 \%$. La densidad poblacional máxima se registró el 22 de abril al alcanzar 13 individuos a $20,12^{\circ} \mathrm{C}$ de temperatura y humedad relativa de $86,77 \%$.

Respecto a la variable grado de infestación también de acuerdo a la Figura 6, el menor grado registrado fue el grado 1 con 0 individuos, y el máximo fue de grado 4 en la evaluación del 22 de abril con 13 individuos registrados. La infestación fue variable durante el desarrollo del cultivo, relacionado directamente a las aplicaciones del insecticida imidacloprid.

\section{Bemisia tabaci (Gennadius)}

Esta plaga se registra desde el 28 de enero con 2 adultos por 100 brotes evaluados en dicha evaluación, a $23,3^{\circ} \mathrm{C}$ y humedad relativa de $75,14 \%$ como puede apreciarse en la Figura 7.

Las ocurrencias más altas se registraron en las evaluaciones del 04 y del 25 de marzo registrando 3 adultos por 100 brotes en ambos casos a $22,66^{\circ} \mathrm{C} \mathrm{y}$ $83,68 \%$ de humedad relativa y $25,38^{\circ} \mathrm{C}$ y humedad relativa de $74,37 \%$ respectivamente.

\section{Spodoptera ochrea (Hampson)}

Se reportó 1 masa de huevos en las evaluaciones del 21 de enero y del 04 de febrero, a $20,62^{\circ} \mathrm{C}$ y $86,12 \%$ de humedad relativa la primera, y $22,96^{\circ} \mathrm{C}$ y humedad relativa de $84,5 \%$ en la segunda; representando los puntos más bajos de esta plaga con respecto a la variable número de masas de huevos. Dicha variable se incrementó en abril alcanzando el máximo de 10 masas de huevos reportadas en la evaluación del 15 de abril a $23,3^{\circ} \mathrm{C}$ y humedad relativa de $78,33 \%$ como puede apreciarse en la Figura 8.

La oviposición de masas de huevos posiblemente tiene relación con el traslado de individuos adultos desde el campo colindante también de sandía. Asimismo, en los alrededores del campo evaluado, se detectó la presencia de Amaranthus dubius, maleza hospedera de Spodoptera ochrea. 

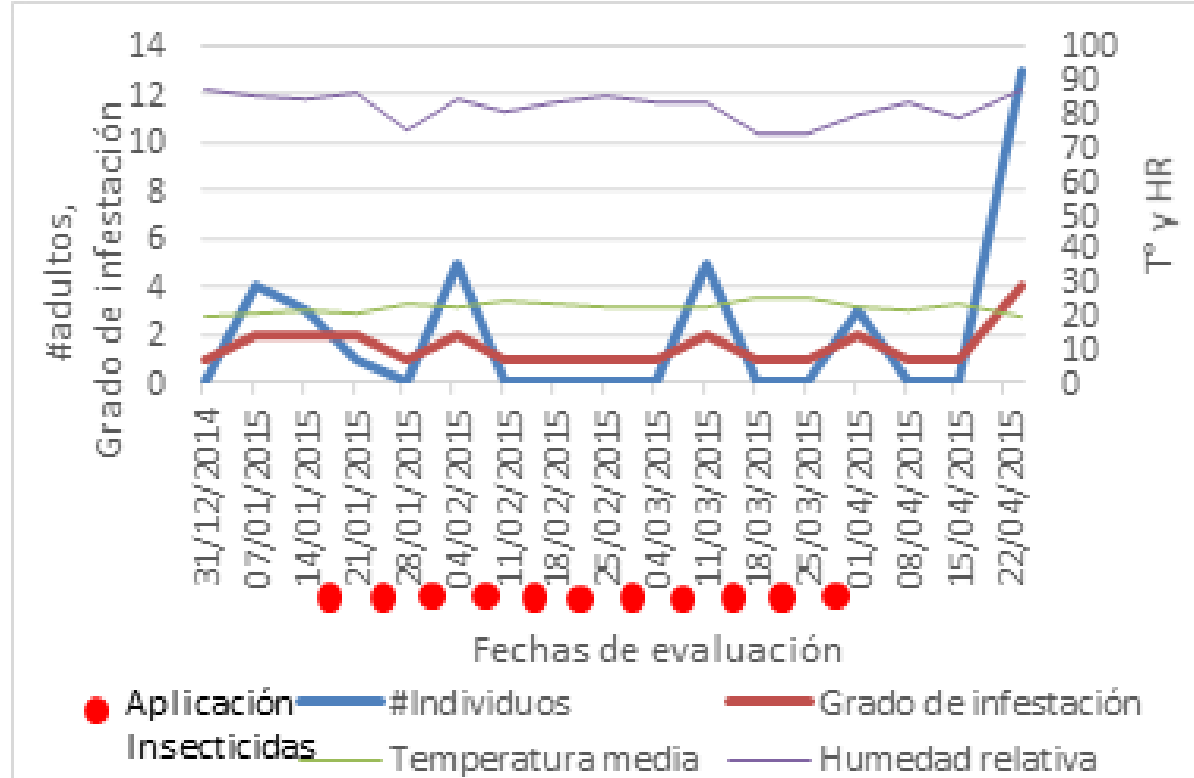

Figura 6. Ocurrencia estacional de adultos de Aphis gossypii (Glover)y grados de infestación en sandía, variedad Santa Amelia. Periodo:diciembre 2014 - abril 2015. La Molina, Lima - Perú.

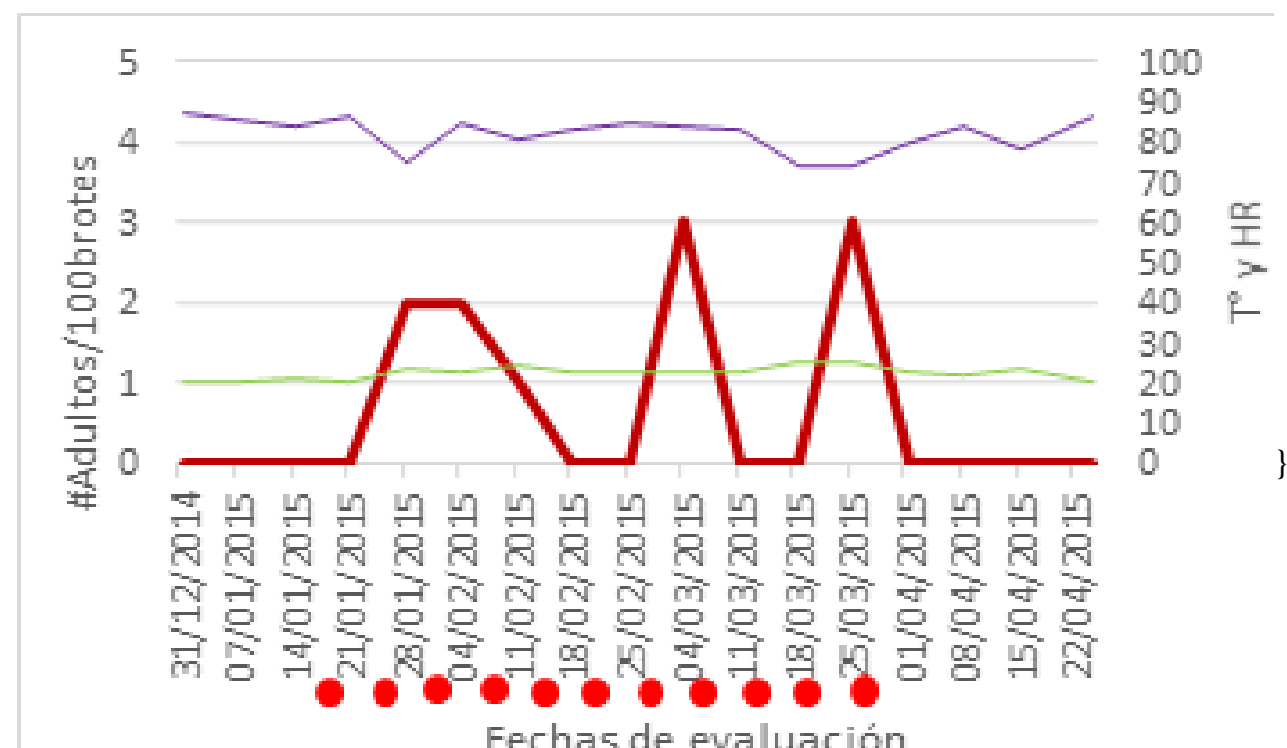

Fechas de evaluación

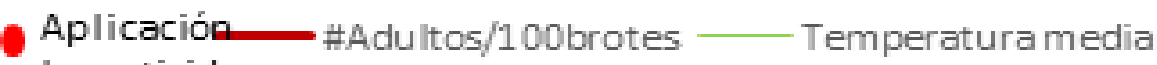
Insecticidas

Figura 7. Ocurrencia estacional de adultos de Bemisia tabaco (Gennadius) en 100 brotes de sandía, variedad Santa Amelia. Periodo: diciembre 2014 - abril 2015. La Molina, Lima - Perú. 


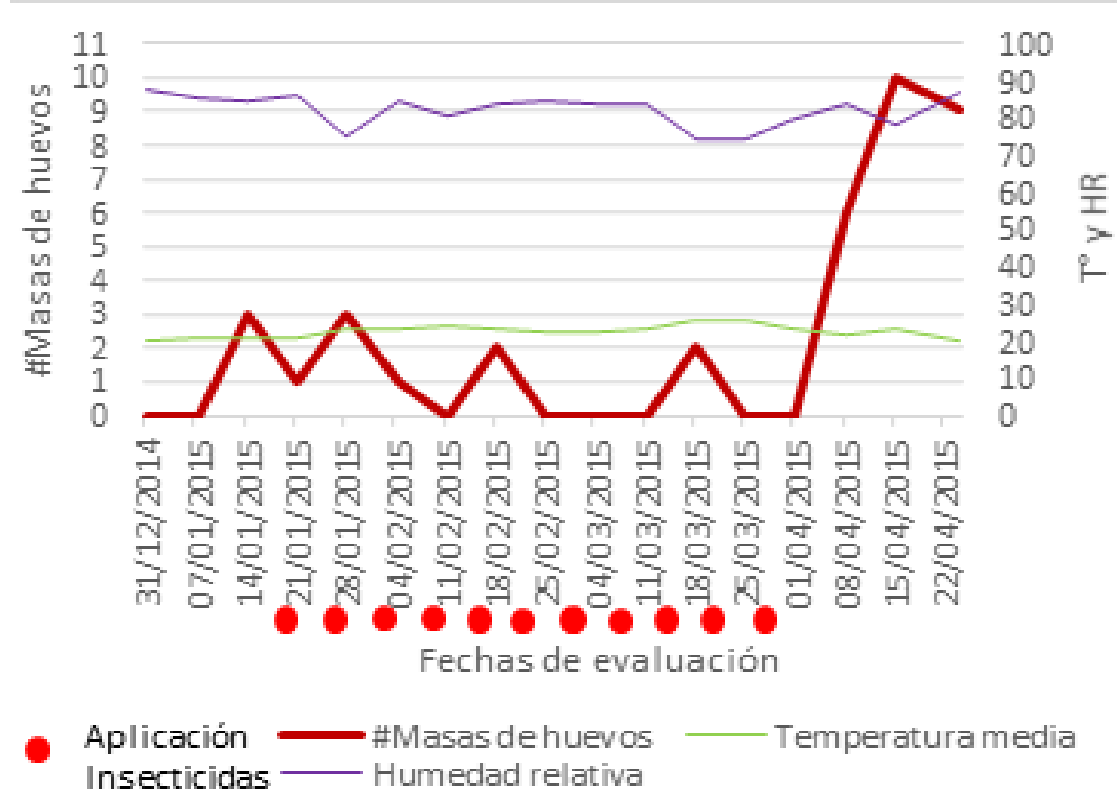

Figura 8. Ocurrencia estacional de masas de huevos de Spodopteraochrea (Hampson) en hojas de sandía, variedad Santa Amelia. Periodo: diciembre 2014 - abril 2015. La Molina, Lima - Perú.

Respecto a la variable número de larvas, la plaga se registra a partir de la evaluación del 7 de enero presentando 8 larvas a $20,58^{\circ} \mathrm{C}$ de temperatura y humedad relativa de $85,52 \%$.

En adelante la ocurrencia de larvas es variable, con subidas y bajadas que coinciden con las aplicaciones de insecticidas para después dispararse registrando 389 larvas a $22,03^{\circ} \mathrm{C}$ y humedad relativa de $83,62 \%$ en la evaluación del 8 de abril, alcanzando el máximo el 15 de abril con 698 larvas con $23,3^{\circ} \mathrm{C}$ de temperatura y humedad relativa de $78,33 \%$.

Sobre el particular, Casana y Vergara (2006) afirman que el género Spodoptera es la plaga más frecuente en costa y que puede causar daños muy severos al área foliar de no controlarse oportunamente. Esto coincide con lo que se aprecia en la Figura 9, en la que puede observarse que la presencia de larvas de Spodoptera ochrea se reportó en casi todas las evaluaciones realizadas, estas causaron daños importantes al alimentarse de las hojas del cultivo.

A su vez, Spodoptera ochrea comenzó a infestar flores a partir de la evaluación del 28 de enero registrándose una flor infestada a $23,3^{\circ} \mathrm{C}$ y humedad relativa de $75,14 \%$, lo que coincidió con los primeros días de floración ,y su presencia se mantuvo en flores hasta el 22 de abril. El punto máximo en la variable número de flores infestas fue de nueve en la evaluación del 15 de abril a $23,3^{\circ} \mathrm{C}$ y humedad relativa de $78,33 \%$ tal como puede apreciarse en la Figura 10.

En cuanto a la variable número de frutos infestados, se reportó a partir de la evaluación del 11 de febrero registrando siete frutos infestados a una temperatura de $24,41^{\circ} \mathrm{C}$ y humedad relativa de $80,75 \%$, alcanzando el punto más alto en la evaluación del 01 de abril registrando 60 frutos infestados a $22,95^{\circ} \mathrm{C}$ de temperatura y humedad relativa de $79,68 \%$.

\section{Prodiplosis longifila (Gagné)}

Solo se presentó en dos evaluaciones después del trasplante, con dos brotes infestados el 14 de enero a $21,16^{\circ} \mathrm{C}$ y humedad relativa de $84,31 \%$; y con 3 brotes infestados el 21 de enero a una temperatura de $20,62^{\circ} \mathrm{C}$ y humedad relativa de $86,12 \%$ como puede apreciarse en la Figura 11 

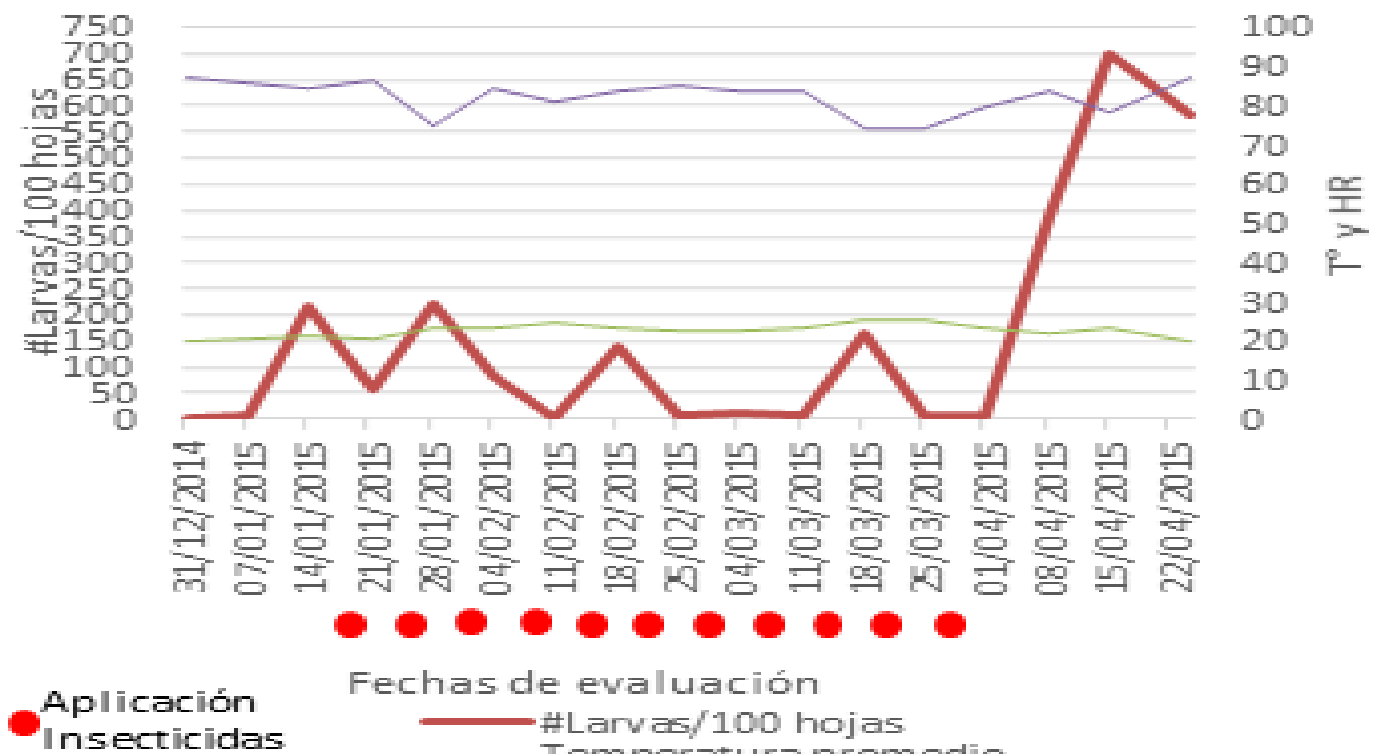

Fechas de evaluación
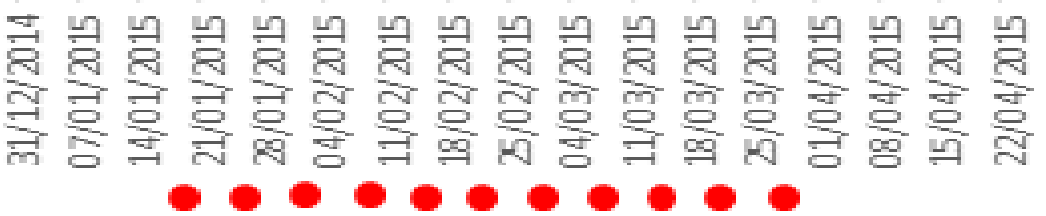

Insecticidas

\#Larvas/100 hojas

Temperatura promedio

Humedad relativa

Figura 9. Ocurrencia estacional de larvas de Spodoptera ochrea (Hampson) en hojas de sandía, variedad Santa Amelia.Periodo: diciembre 2014 - abril 2015. La Molina, Lima - Perú.
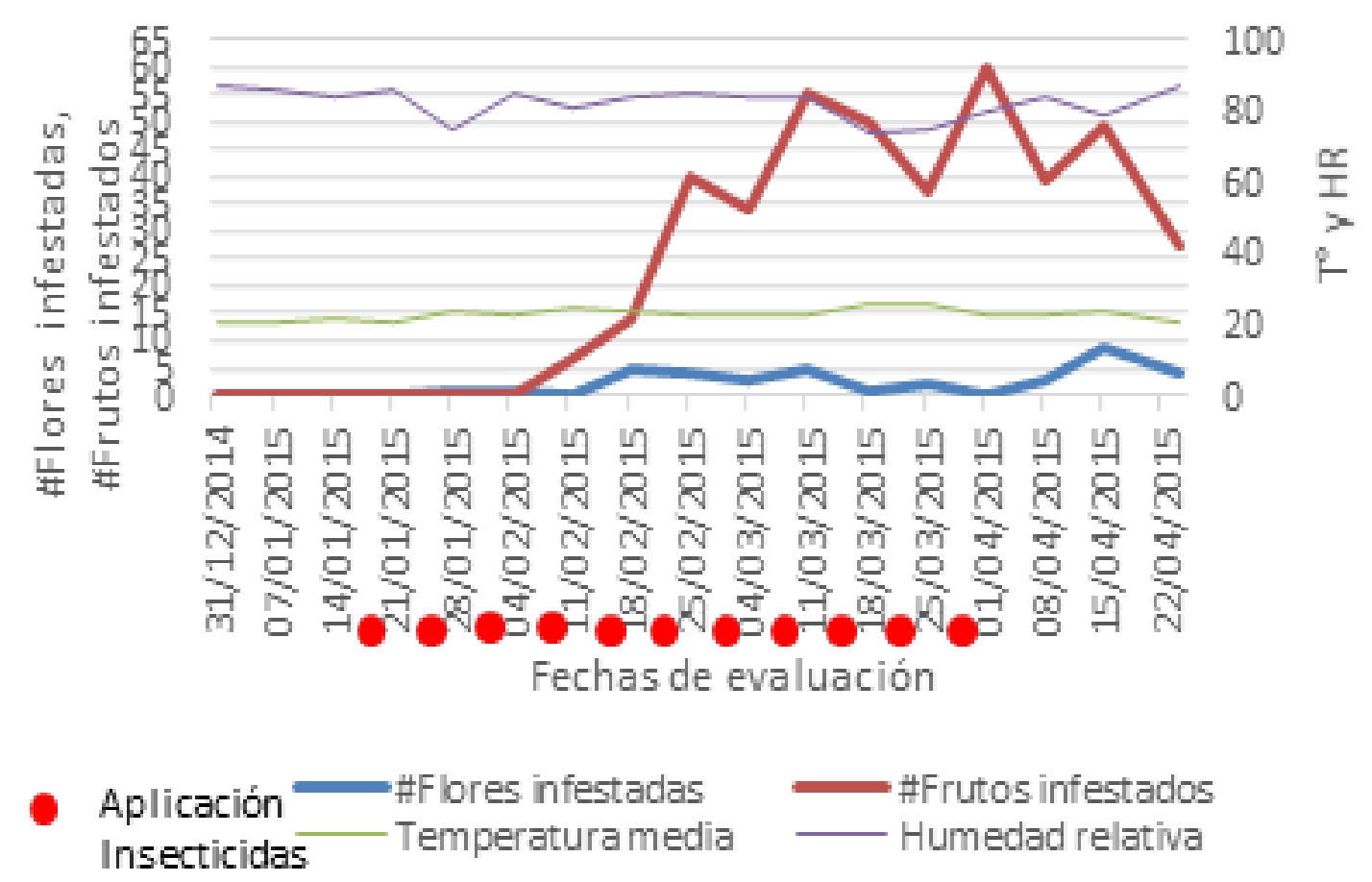

Figura 10. Número de flores y frutos infestados por larvas de Spodoptera ochrea (Hampson) en sandía, variedad Santa Amelia. Periodo: diciembre 2014 - abril 2015. La Molina, Lima - Perú. 


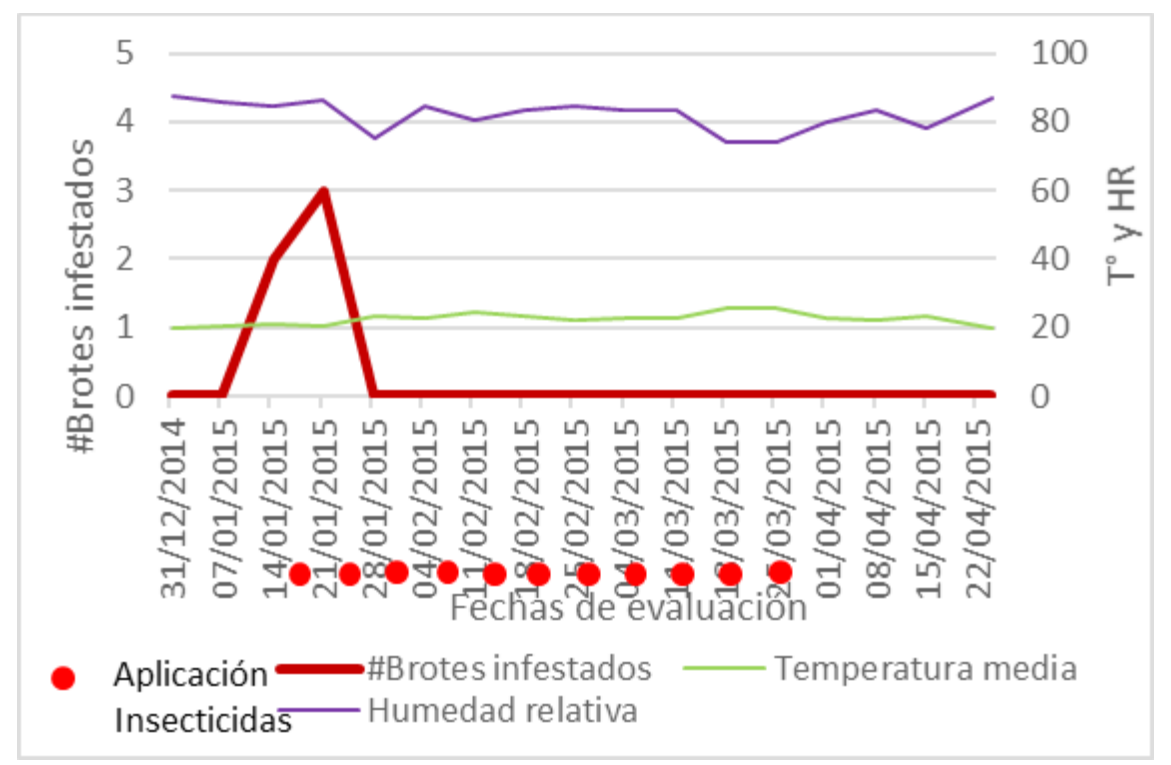

Figura 11. Número de brotes infestados por larvas de Prodiplosislongifila (Gagné) en sandía, variedad Santa Amelia. Periodo:diciembre 2014 - abril 2015. La Molina, Lima-Perú.

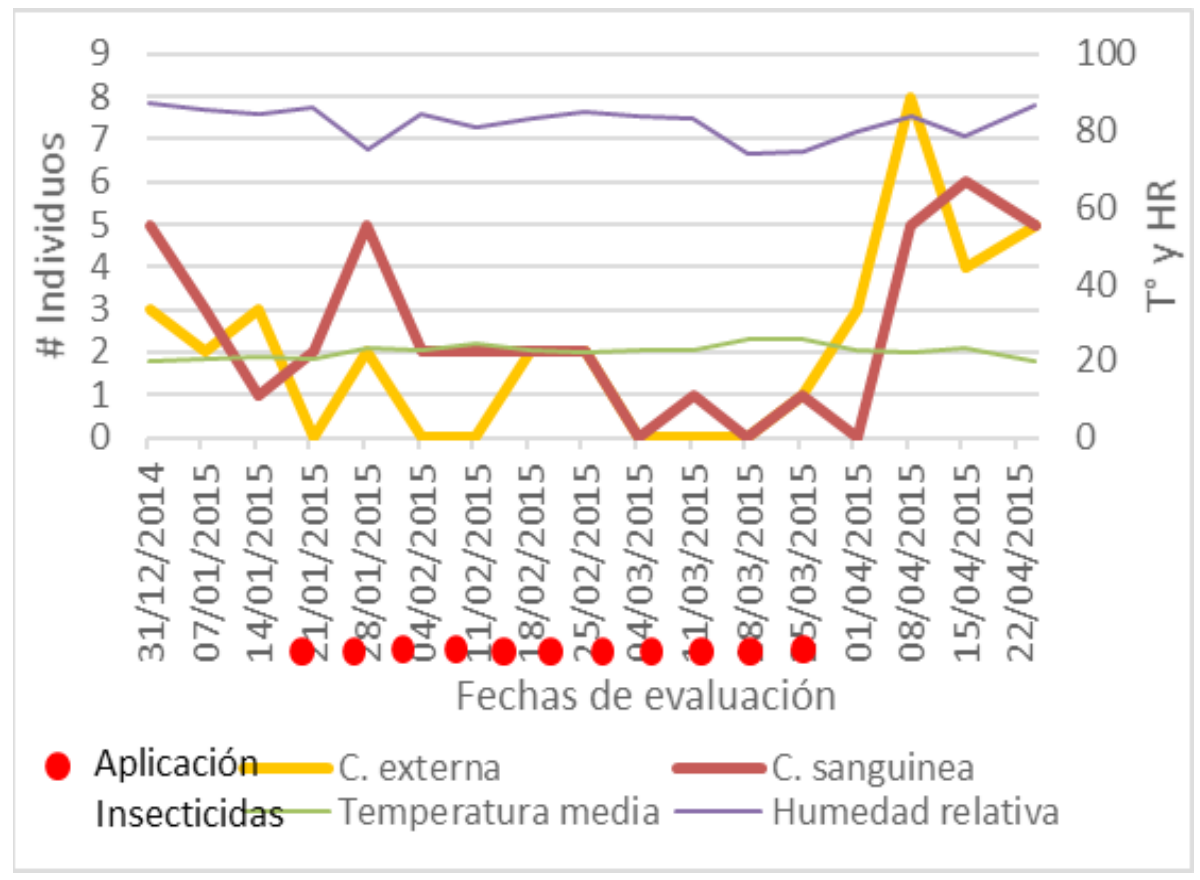

Figura 12. Ocurrencia estacional de algunos predadores registradosen el cultivo de sandía, variedad Santa Amelia. Periodo: Diciembre2014 - Abril 2015. La Molina, Lima - Perú. 
Probablemente, la baja infestación de Prodiplosis longifila se debió a las continuas aplicaciones del insecticida fipronil.

\section{Predadores de plagas de sandía}

Los enemigos naturales registrados en el campo de sandía fueron: Chrysoperla externa (Hagen) y Cycloneda sanguinea (Linnaeus).

La menor cantidad del controlador Chrysoperla externa se registró en la evaluación del 25 de marzo con un solo individuo a $25,38^{\circ} \mathrm{C}$ de temperatura y humedad relativa de $74,37 \%$. Y a su vez, la presencia más alta de dicho predador fue en la evaluación del 08 de abril registrando 8 individuos a una temperatura de $22,03^{\circ} \mathrm{C}$ y humedad relativa de $83,62 \%$ como puede apreciarse en la Figura 12.

Las menores apariciones de Cycloneda sanguinea se dieron en las evaluaciones del 14 de enero y del 25 de marzo con un solo individuo a $21,16^{\circ} \mathrm{C}$ y $25,38^{\circ} \mathrm{C}$ de temperatura respectivamente. Asimismo, la mayor cantidad de este predador tuvo lugar en la evaluación del 15 de abril al registrar 6 individuos a una temperatura de $23,3^{\circ} \mathrm{C}$ y humedad relativa de $78,33 \%$. Los adultos de Chrysoperla externa (Hagen) y Cycloneda sanguinea (Linnaeus) se presentaron principalmente en las hojas y no fueron muy abundantes debido a su exposición a la aplicación de insecticidas, lo que disminuyó su densidad poblacional.

\section{CONCLUSIONES}

Agrotis ipsilon (Hufn.) presenta población de larvas las dos primeras semanas después trasplantar los plantines de sandía. Por otro lado, Diaphania nitidalis (Stoll) estuvo presente desde la etapa de desarrollo vegetativo hasta fructificación.Spodoptera ochrea (Hampson) fue la plaga principal y sus larvas se registraron durante todo el ciclo del cultivo, desde desarrollo vegetativo a fructificación. Con respecto a enemigos naturales, Chrysoperla externa (Hagen) y Cycloneda sanguinea (Linnaeus) fueron los predadores registrados. Las plagas Bemisia tabaci (Gennadius), Aphis gossypii (Glover), Liriomyza huidobrensis (Blanchard) y Prodiplosis longifila
(Gagné) registraron bajos niveles poblaciones, tanto en desarrollo vegetativo, floración y fructificación. Finalmente, se sugiere el estudio de ocurrencia estacional de plagas de otros cultivos importantes en diferentes regiones del país.

\section{Conflictos de intereses}

Los autores firmantes del presente trabajo de investigación declaran no tener ningún potencial conflicto de interés personal o económico con otras personas $\mathrm{u}$ organizaciones que puedan influir indebidamente con el presente manuscrito.

\section{Contribuciones de los autores}

Preparación y ejecución: DC, GJ, JC. AB, PR; Desarrollo de la metodología: DC, GJ, JC. AB, PR; Concepción y diseño: DC, GJ, JC. AB, PR; Edición del artículo: DC, GJ, JC. AB, PR; Supervisión del estudio: DC, GJ, JC. AB, PR.

\section{LITERATURA CITADA}

- Casana, N., \& Vergara, C. (2006). Biología y comportamiento de Spodoptera ochrea (Hampson) (Lepidóptera: Noctuidae) en el cultivo de espárrago (Asparagus officinalis L.), bajo condiciones de laboratorio. Rev. Per. Ent., 45: 141-143.

- Escalona, V., Alvarado, P., Monardes, H., Urbina, C., \& Martin, A. (2009). Manual de Cultivo de sandía (Citrullus lanatus) y melón (Cucumis melo L.). Facultad de Ciencias Agronómicas de la Universidad de Chile. 18 p.

- Goreta, S., Perica, S., Dumiicic, G., Bucan, L., \& Zanic, K. (2005). Growth and Yield of Watermelon on Polyethylene Mulch with different Spacings and Nitrogen Rates. J. Armer. Soc. Hort. Sci., 40:366-369.

- Guner, N., \& Wehner, TC. (2004). The Genes of Water melon. J. Amer.Soc.Hort.Sci., 39: 11751182 .

- Huh, C., Solmaz, I., \& Sari, N. (2008). Caracterización morfológica de germoplasma de sandía en Korea y Turquía. Procedimiento de la IX $^{\text {th }}$ EUCARPIA reunión sobre la genética de las 
cucurbitáceas INRA, Avignon. France. May $21^{\text {st }}$ $24^{\text {th }}$.

- Mujica, N., \& Cisneros, F. (1995). Developing IPM components for Leafminer Fly in Cañete, Valley of Perú. En: International Potato Center Program Report 1995-96. CIP, Lima, Perú, 177184.

- Sánchez, G., \& Vergara, C. (2003). Manual de Prácticas de Entomología Agrícola. Universidad Nacional Agraria La Molina. Departamento de Entomología. $5^{a}$ ed.Lima, Perú, 172 p.

- Zarate, C. (1972). Control químico del perforador de guías y frutos de las cucurbitáceas Diaphania nitidalis $\mathrm{S}$. en pepinillo.Tesis para optar título de Ingeniero Agrónomo. Universidad Nacional Agraria La Molina.122 p.

- Zohary, D.; \& Hopf, M. (2000). Domestication of Plants in the Old-World $3^{\text {rd }}$ edition. Oxford University Press. 193 p. 